\title{
Optimization of Engine Room Structure under Static and Dynamic Constraints Using Genetic Algorithms
}

\author{
by Hisashi Nobukawa*, Member \\ Mitsuru Kitamura*, Member
}

\author{
Fengxiang Yang**, Student Member \\ Guoqiang Zhou*, Member
}

\begin{abstract}
In this paper, the Genetic Algorithm is used to optimize the engine room structure under the static and dynamic constraints. A penalty function method is proposed to handle the complicated constraint conditions which include stress, natural frequency, and allowable magnitude of design variables. The new operator called objective elitism as well as fitness elitism is introduced to improve the efficiency of the method. Varied probability of mutation is proposed to improve the convergence of the algorithm without the risk of premature convergence to a poor solution. To handle the discrete problems, we introduced the serial numbers of cross $^{-}$sections whose shape data are stored in database beforehand. Successful application of the Genetic Algorithm in the engine room optimization suggests its use to more complex engineering design problems.
\end{abstract}

\section{Introduction}

Optimization of engine room structure under the static and dynamic constraints is found to be complex because of the implicit characteristics of constraints. There are some cases that the static strength conditions are satisfied, but the engine room structure undergoes large-amplitude vibration because the natural frequencies of the engine room structure are close to the exciting frequency.

Another problem ${ }^{4)}$ in practice design is the discrete design variables. In ship building designs, the shape steel members are used commonly and their crosssections vary discretely. The formulae to represent all the cross-sections can not be formed because they vary irregularly. It is difficult for traditional optimization techniques to handle this problem. In this study, we focus our study on these two problems.

Genetic Algorithms (GAs) ${ }^{3), 5), 8)}$ are powerful and broadly applicable stochastic search and optimization techniques based on principle from evolution theory. Recently, Genetic Algorithms have received considerable attention regarding their potential as a novel optimization technique ${ }^{1,2)}$. By using Genetic Algorithms, the global optimum can be reached more easily than some traditional optimization techniques. One of other major advantages is that they can be applied to the optimization problems with discrete design variables by

\footnotetext{
* Faculty of Engineering, Hiroshima University

** Graduate School, Hiroshima University
}

Received 9th Jan. 1998

Read at the Spring meeting 14, 15th May 1998 introducing the serial numbers of shape steel members whose shape data of cross-sections are stored in database beforehand.

In this study, A penalty function method is proposed to handle the complicated constraint conditions which are the implicit functions of the design variables. Besides the fitness elitism, the objective elitism is proposed for the first time. Results demonstrate that the introduction of objective elitism improves the GA in this problem significantly. Varied probability of mutation is also introduced to speed the convergence to the global optimal point. To handle the discrete problem, the serial numbers of cross- $^{-}$sections are introduced to be design variables which are corresponding to the certain sizes of cross $^{-}$sections.

\section{Problem Statement}

In this study, the objective is to find the design variables $\boldsymbol{X}=\left(x_{1}, x_{2}, \cdots, x_{q}\right)$ to minimize the construction cost of the engine room structure under the static and dynamic constraints as the following :

$\min f(\boldsymbol{X})$

subject to the constraints as below :

(1) Static bending and shear stress constraints for the web frames, web beams and longitudinal girders

$$
\sigma_{i, \max }(\boldsymbol{X}) \leq \sigma_{i, 0} \quad i=1,2, \cdots, n
$$

Here $\sigma_{i, \max }(\boldsymbol{X})$ is the maximum of the stress in element $i$. $\quad \sigma_{i, 0}$ is the maximum allowable stress for the $i$-th element.

$$
\begin{aligned}
& \text { (2) Dynamic constraints } \\
& \xi_{i}(\boldsymbol{X}) \geq \omega_{0}^{2} \text { for all } i \\
& \xi_{i}(\boldsymbol{X}) \leq \omega_{1}^{2} \text { for the first } I \text { eigenvalues } \\
& \xi_{i}(\boldsymbol{X}) \geq \omega_{2}^{2} \text { for all } i>I
\end{aligned}
$$

where $\omega_{j}^{2}$ are the given bounds of $\xi_{i}$. It is desirable to 


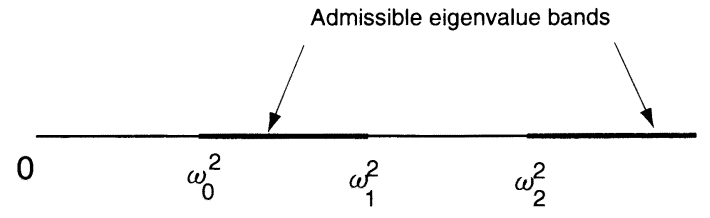

Fig. 1 Graphical representation of frequency constraints

design the engine room structure so that no natural frequencies lie within the band $\left[\omega_{1}, \omega_{2}\right]$ because the exciting frequency of the main engine falls in this band. $\omega_{1}, \omega_{2}$ can be determined according to the exciting frequency. Graphically, the constraints of formulae (3) to (5) require that the eigenvalues lie in the admissible bands of Fig. 1. $\omega_{0}$ is the low limit of the constrained frequency.

(3) Design variable constraints

From economic and manufacturing points of view, it is desirable to put upper and lower bound on design variables as below:

$$
x_{i, \min } \leq x_{i} \leq x_{i, \max } \quad i=1,2, \cdots, q
$$

$\sigma_{i, \max }(\boldsymbol{X})$ in formula 2 can be obtained by solving the structural static equilibrium equation ( 7 ) below.

$$
\boldsymbol{K}(\boldsymbol{X}) \boldsymbol{Y}=\boldsymbol{P}
$$

$\xi_{i}(\boldsymbol{X})$ in formulae ( 3 ) to ( 5 ) is calculated out by solving the eigenvalue problem as below:

$$
\boldsymbol{K}(\boldsymbol{X}) \boldsymbol{U}=\xi_{i} \boldsymbol{M}(\boldsymbol{X}) \boldsymbol{U}
$$

where $\boldsymbol{M}(\boldsymbol{X})$ is the mass matrix. $\quad \xi_{i}$ is the $i$-th eigenvalue. $\boldsymbol{U}$ is the matrix of eigenvetors.

\section{Design variables}

Design variables include the cross-sectional sizes of web frame and web beam members, web frame spacings and the hull thickness shown in Fig. 2.

In practical ship design, some variables vary discretely. Usually designers can select the web frames and the web beams from a certain limited number of standard shape steel members available commercially like the ones shown in Table 1 . Obviously these kinds of variables vary discretely, irregularly and should be represented by four parameters $t_{0}, t_{1}, b, h$ shown in Fig. 2. However, the four parameters representing the cross -section can not be taken as the design variables independently because they can not be combined arbitrarily without considering the availability in market. Therefore, the cross-section represented by four parameters should be taken as one design variable. In order to achieve this goal, the serial numbers of standard shape steel members are introduced as the design variables. Once a serial number is fixed, the corresponding cross-section sizes can be gotten through the database stored in computer beforehand.

The web frame spacings are also taken as the continuous design variables in this study shown in Fig. 2. design variables (one chromosome)

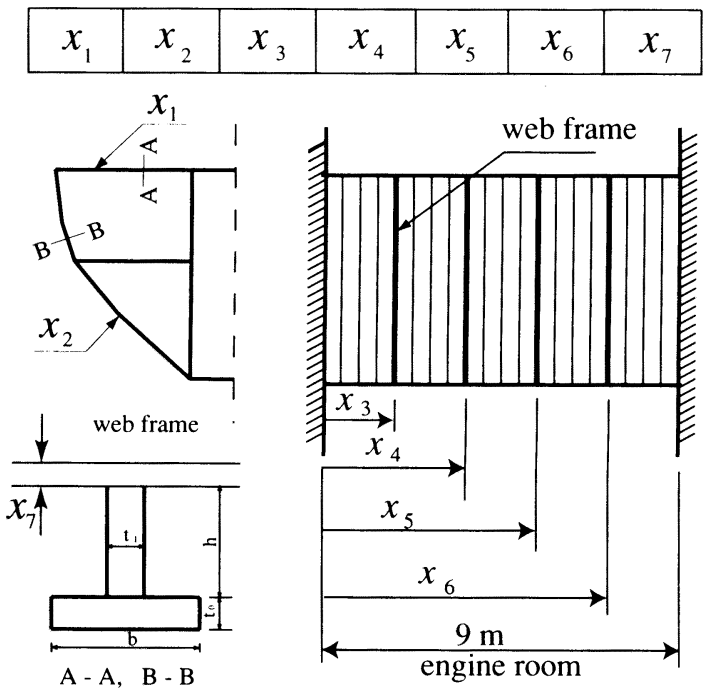

Fig. 2 Graphical representation of variabless

Table 1 Serial Number and corresponding cross-section

\begin{tabular}{|l|l|l|l|}
\hline serial & \multicolumn{1}{|c|}{$\square$} & serial & $\square$ \\
\hline No & ${\mathrm{h} \times \mathrm{b} \times \mathrm{t}_{1} / \mathrm{t}_{0}}$ & $\mathrm{No}$ & $\mathrm{h} \times \mathrm{b} \times \mathrm{t}_{1} / \mathrm{t}_{0}$ \\
\hline 1 & $200 \times 120 \times 8 / 8$ & 17 & $400 \times 200 \times 14 / 12$ \\
2 & $200 \times 125 \times 12 / 8$ & 18 & $400 \times 250 \times 14 / 12$ \\
3 & $200 \times 150 \times 12 / 8$ & 19 & $400 \times 300 \times 14 / 10$ \\
4 & $250 \times 200 \times 12 / 8$ & 20 & $400 \times 300 \times 14 / 12$ \\
5 & $300 \times 125 \times 12 / 10$ & 21 & $500 \times 200 \times 12 / 10$ \\
6 & $300 \times 150 \times 12 / 10$ & 22 & $500 \times 200 \times 15 / 12$ \\
7 & $300 \times 200 \times 12 / 10$ & 23 & $500 \times 250 \times 12 / 10$ \\
8 & $300 \times 200 \times 12 / 12$ & 24 & $500 \times 250 \times 15 / 12$ \\
9 & $350 \times 150 \times 12 / 10$ & 25 & $500 \times 300 \times 15 / 12$ \\
10 & $350 \times 150 \times 12 / 12$ & 26 & $550 \times 200 \times 16 / 11$ \\
11 & $350 \times 200 \times 12 / 10$ & 27 & $550 \times 250 \times 16 / 12$ \\
12 & $350 \times 200 \times 12 / 12$ & 28 & $550 \times 300 \times 16 / 12$ \\
13 & $350 \times 250 \times 12 / 12$ & 29 & $550 \times 400 \times 16 / 12$ \\
14 & $400 \times 150 \times 10 / 10$ & 30 & $600 \times 200 \times 16 / 11$ \\
15 & $400 \times 150 \times 10 / 12$ & 31 & $600 \times 300 \times 16 / 11$ \\
16 & $400 \times 200 \times 12 / 12$ & 32 & $600 \times 400 \times 16 / 16$ \\
\hline
\end{tabular}

\section{Genetic Algorithms}

Genetic Algorithms ${ }^{3,55,8)}$ are powerful tools for solving the optimization problems especially the hard problems $^{7}$. Quite different from the traditional optimization techniques, GAs work on a population of candidate designs and the candidates compete to contribute to the production of new designs according to their fitness. Each candidate is represented by a string of bits which is a coded listing of the values of the design 


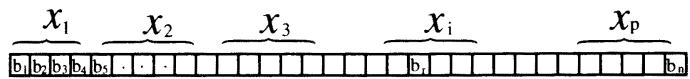

one chromosome

Fig. 3 Graphical representation of variables

variables shown in Fig. 3. The fitter substrings will survive during the reproduction operation, and be improved further through the crossover and mutation operation. With the process repeating over many generations, the best design can be found.

\section{1 Constraint handling}

Most real problems of function optimization involve constraints. In GAs, constraint handling ${ }^{5)}$ can be done by penalty methods ${ }^{9)}$ which use penalty functions as an adjustment to the optimized objective function. Therefore, a constrained problem is transformed to an unconstrained problem by associating a penalty with all constraint violations. Thus formula ( 1 ) above is transformed into optimization of the function:

$$
F(\boldsymbol{X})=f(\boldsymbol{X})+\delta \sum_{i=1}^{p} \Phi_{i}(\boldsymbol{X})
$$

where $p$ is the total number of constraints, $\delta$ is a penalty coefficient, $\Phi_{i}(\boldsymbol{X})$ is a penalty term related to the $i$-th constraint $(i=1, \cdots, p) . \delta$ is a scalar multiplier intended to control the penalty imposed in considering points that violate constraints. if $\delta$ is small, then the algorith$m$ will allow much exploration of constraint violation space and if $\delta$ is large, very few points that violate constraints will survive. The determination of $\delta$ value should be considered with the term $\sum_{i=1}^{p} \Phi_{i}(\boldsymbol{X})$ together.

There are a variety of possible penalty functions which can be applied. For the constraint $g_{i}(\boldsymbol{X}) \leq 0$, the penalty function can be written as the following form :

$$
\Phi_{i}(\boldsymbol{X})= \begin{cases}0 & g_{i}(\boldsymbol{X}) \leq 0 \\ \left|g_{i}(\boldsymbol{X})\right|^{m} & g_{i}(\boldsymbol{X})>0\end{cases}
$$

In practical applications, $m$ can be $0,1,2$. When $m=$ 0 , any penalty term is assigned a constant regardless their degrees of violation. The other penalty functions depend on the degree of violation: the larger the violation is, the greater the penalty is imposed. In this study, the growth of the penalty function is quadratic $(m=2)$ with respect to the degree of violation.

Generally speaking, the objective term and the penalty term should be in the same order of figure. If the objective is too large compared with the penalty term, the process of the optimization will drive all the chromosomes into the infeasible domain. Otherwise if the penalty terms are designed much larger than the objective, the selection pressure will become very high, as a result, a few super chromosomes will dominate the selection process, this will result in premature.

\section{2 Fitness function and objective function}

A minimum problem like formula ( 9 ) can be transformed to the maximum problem by adding the negative sign. By doing this, the optimization function will return negative values. Because Genetic Algorithms are on the assumption that the fitness function returns only positive values, therefore, a constant $C$ is added to satisfy the requirement. Consequently the fitness function can be formed as below :

$$
V(\boldsymbol{X})=C-f(\boldsymbol{X})-\delta \sum_{i=1}^{p} \Phi_{i}(\boldsymbol{X})
$$

Here $f(\boldsymbol{X})$ is the objective function and $V(\boldsymbol{X})$ is the fitness function. $C$ should be selected as small as possible on condition that $V(\boldsymbol{X})>0$. If $C \gg F(\boldsymbol{X})$,the function $V(\boldsymbol{X})$ will suffer from much slower convergence than the function $F(\boldsymbol{X})$. In the extreme case, the $V(\boldsymbol{X})$ will be optimized using a totally random search; conversely, $V(\boldsymbol{X})$ may be converging too fast, pushing the algorithm into a local optimum. In this study, because the largest value of the objective without considering the static and dynamic constraints can be calculated beforehand, and the largest penalty term can be also known in advance according to experiences, $C$ can be fixed by summing the largest values of them.

\section{3 Selection and elitism}

In this study, selection is used to form the next generation with a probabilistic mechanism. Each chromosome receives the number of its offspring based on its survival probability. However, in the application to the engine room optimization, the traditional simple GAs have two drawbacks. Firstly because the offspring in each generation replace their parents soon after they are born, they may lose the best chromosomes of the old generation. Quite often, the optimum fitness of later generation is less than that of the generation ahead, especially when the cost value near the global optimal value, This lead to the slow convergence to the final optimal value. As is known to all, dynamic analyses of structures are the heavy task in optimization process, these methods are time-consuming. Secondly it is difficult to know when the optimal point is found out because after several generations, the values of individuals oscillate taking larger and smaller values over generations. In order to solve these problems, elitist selection is introduced to ensure that the best chromosome is passed into the new generation if it is not selected through another process of selection. The best chromosomes in a generation are selected according to their fitness and objective values in infeasible and feasible domain respectively. In this study, the objective elitist technique is introduced because minimizing the objective is the real goal. This operator improves GAs significantly and makes us set the termination condition easily.

\section{4 Crossover and Mutation}

Crossover operates on two chromosomes at a time and generates offspring by combining both chromosome's features. A higher crossover rate allows exploration of more of the solution space and reduces the chances of settling for a false optimum; but if this rate is too high, it results in the wastage of a lot of computation time in exploring unpromising regions of 
the solution space. Mutation arbitrarily alters one or more genes of a selected chromosome by a random change with the mutation rate. This operation keeps the chromosomes to be diversity to prevent the searching from premature. The mutation rate is usually held constant throughout the calculation of the Genetic Algorithm. However, this does not work very efficiently because at the beginning of a run of Genetic Algorithms, the exploration of the search space is needed. Therefore, the mutation rate should be higher at the start of the run. But after the promising regions of the search space are obtained, the exploitation becomes more important and the mutation rate should be lower than before. Therefor the mutation rate is proposed as below :

$$
p_{m}^{(n+1)}=\lambda^{n} p_{m}^{(n)}
$$

where $\lambda$ is the decreasing rate coefficient which should be less than $1 . \quad n$ is the iteration number. Generally speaking, varying mutation rate can improve the performance of Genetic Algorithms, especially when the high precision is required.

\section{5 Termination condition}

Until now the common way to control the convergence in GAs is to set a fixed generation number. These termination conditions need the knowledge on the characteristic of function. It is quite difficult to fix the total number of generations before the calculation of a problem. In this study, because the objective elitism is introduced, the termination condition is modified in the following way. Keep track of the objective value $f(t)$ of the best individual in generation $t$, if

$$
|f(t)-f(t+N)| \leq \varepsilon
$$

for the small $\varepsilon$ defined as a constant in the program, terminate the evolutionary loop. $N$ is determined by the precision and the nature of fitness function. Too small $N$ will result in the false convergence, but too large $N$ will increase the computing time. For the numerical example, $N=30$ is suggested.

\section{Optimization procedures} tion.

Step 1. Create an initial population of potential solu-

Step 2. Calculate the maximum stresses of structure according to Eq. 7 ).

Step 3. Calculate the first $N$ eigenvalues of structure according to Eq. ( 8 ).

Step 4. Calculate the penalty terms according to formula (10).

Step 5. Calculate the fitness value $V_{i}$ for each chromosome according to formula (11).

Step 6. Calculate the total fitness of the population

$$
W=\sum_{i=1}^{p o p-s i z e} V_{i}
$$

Step 7. Calculate the probability of a selection for each chromosome

$$
p_{i}=\frac{V_{i}}{W}
$$

Step 8. Calculate the cumulative probability for each chromosome

$$
q_{i}=\sum_{j=1}^{i} p_{j}
$$

Step 9. Generate a random number $r$ from the range $[0,1]$.

Step 10. If $r<q_{1}$ then select the first chromosome; otherwise select the $i$-th chromosome such that $q_{i-1}<r$ $\leq q_{i}$ as the offspring. Circulate step 9 and step 10 for pop-size times.

Step 11. Generate an integer random number $k$ from the range $[1, m-1]$. ( $m$ is the total length of a chromosome).

Step 12. Generate a random number $r^{\prime}$ from the range $[0,1]$.

Step 13. The pair of chromosomes generated in step 10 undergo the crossover operation in the position of $k$ if the random number $r^{\prime}<p_{c}$.

Step 14. Generate a random number $r$ " from the range $[0,1]$.

Step 15. If $r^{\prime \prime}<p_{m}$, mutate the bit. $p_{m}$ is the probability of mutation. Circulate step 14 and step 15 for popsize $\times m$ times.

Step 16. Find out the largest fitness value and the smallest one in infeasible domain.

Step 17. Replace the smallest one in infeasible domains if the largest fitness value in current generation is less than that of previous generation.

Step 18. Find out the smallest objective value and the largest one in feasible domain. If the smallest objective value is larger than that of previous generation, replace the chromosome with the largest objective value by the chromosome of previous generation with the smallest objective value.

Step 19. Go back to step 2 if the termination conditions are not satisfied.

Following selection (step 2 to step 10), crossover (step 11 to step 13), mutation (step 14 and step 15), and elitism (step 16 to step 18), the new population is ready for its next evaluation. So the rest of evolution is just cyclic repetition of the above steps.

\section{Numerical example}

\section{1 Computing model}

The principal dimensions of the ship for numerical example are :

Length P. $\mathrm{P}=70 \mathrm{~m}$; Breadth (mld.) $=12 \mathrm{~m}$;

Depth $(\mathrm{mld})=.7.12 \mathrm{~m}$; Draught $=4.14 \mathrm{~m}$;

Weight of main engine $=31$ tonf.

Weight of generator $=2$ tonf $\times 2$ sets.

The loads imposed upon the ship are shown in Fig. 6. The available standard shape steel members are shown in Table 1. The allowable bending stresses are 18 $\mathrm{kgf} / \mathrm{mm}^{2}$ and $10 \mathrm{kgf} / \mathrm{mm}^{2}$ for the web frame and web beam elements, and longitudinal members respectively. The revolution of main engine with four blades is 284 $\mathrm{rpm}$, therefore the blade frequency is $4.7333 \times 4=$ $18.9333 \mathrm{~Hz}$. To avoid the structural resonance, the 
forbidden frequency band $\left[f_{1}, f_{2}\right]$ is fixed as $[17,21]$.

The simplified three-dimensional finite element model is presented as in Fig. 4 and Fig. 5. In this model, the engine room is modeled into the three dimensional frame in detail, the other part is simplified to be the varying cross-sectional beams with virtual added mass. The numbers of elements and nodes of this model are 408 and 208 respectively. With this model, the natural frequencies of engine room structure can be obtained based on the understanding that the engine room vibration modes are the higher order modes of the computing model, which can be found out by drawing the first $N$ modes of ship, and then selecting the modes in which the vibrations occur mainly in engine room part to be the engine room vibration modes.

\section{2 Design variables}

Design variables include cross-sectional sizes of web

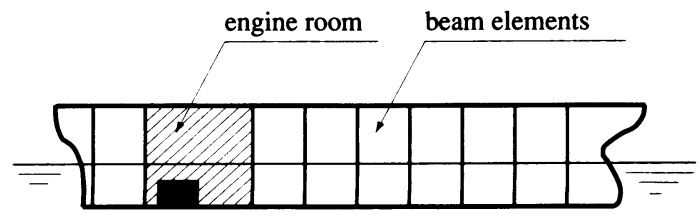

Fig. 4 whole ship structural model

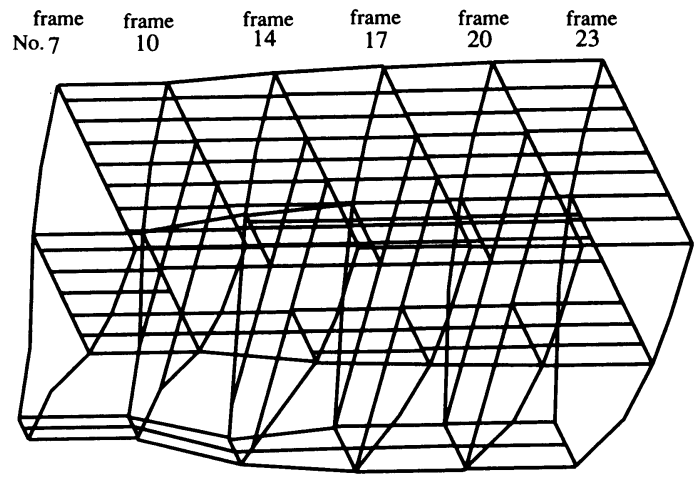

Fig. 5 engine room structural model

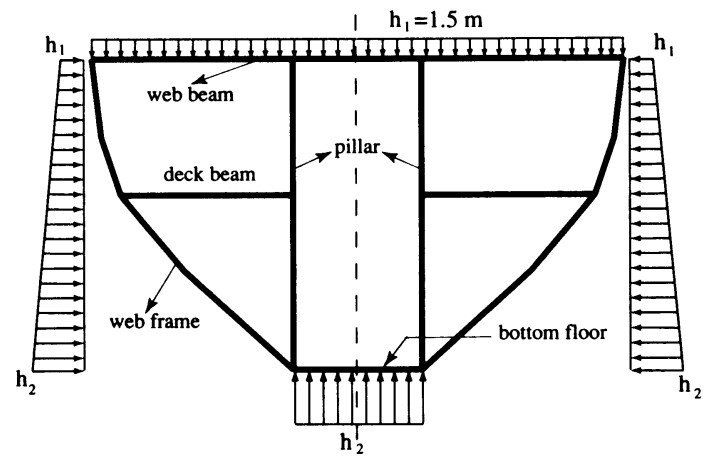

Fig. 6 Loads imposed upon web frame 17 frames and web beams, the web frame spacings and the hull thickness within the engine room part shown in Fig. 2. The cross-sectional information of shape steel members available used in this study is shown in Table 1.

The cross-section of the ship within engine room part varies linearly as in Fig. 7. The unit prices of plate steel and shape steel are 90,000 yens/ton and 105,000 yens/ ton respectively. The price of fillet welding per unit welding length in meter with unit welding leg length in centimeter is 4,200 yens.

\section{3 Numerical tests and discussion}

\subsubsection{Final results of optimization}

Table 2 and curve I in Fig. 8 provide the results of the optimization process on this structure. Here population size $p o p-$ size $=20$, probability of crossover $p_{c}=0.7$, probability of mutation $p_{m}=0.01$. The optimal values are obtained at about generation 43 in which the minimal objective value is $6,000,000$ yens. The optimal crosssectional sizes for the web beams and web frames are $250 \times 200 \times 12 / 8$ and $500 \times 200 \times 12 / 10$ respectively. $x_{i}(i$ $=1, \cdots, 4)$ are $158 \mathrm{~cm}, 356 \mathrm{~cm}, 496 \mathrm{~cm}, 695 \mathrm{~cm}$ respectively. The hull thickness is $10 \mathrm{~mm}$. The maximum stress of transverse elements and longitudinal members are $17.23 \mathrm{kgf} / \mathrm{mm}^{2}$ and $5.031 \mathrm{kgf} / \mathrm{mm}^{2}$ respectively. The natural frequency of engine room structure near the boundary of the dynamic constraints is $21.084 \mathrm{~Hz}$. From the results, it is observed that the values of structural stresses move toward to the boundary of the constraints in general trend, but is not reach the boundary exactly because of the discrete design variables.

6.3.2 Influence of Penalty Term

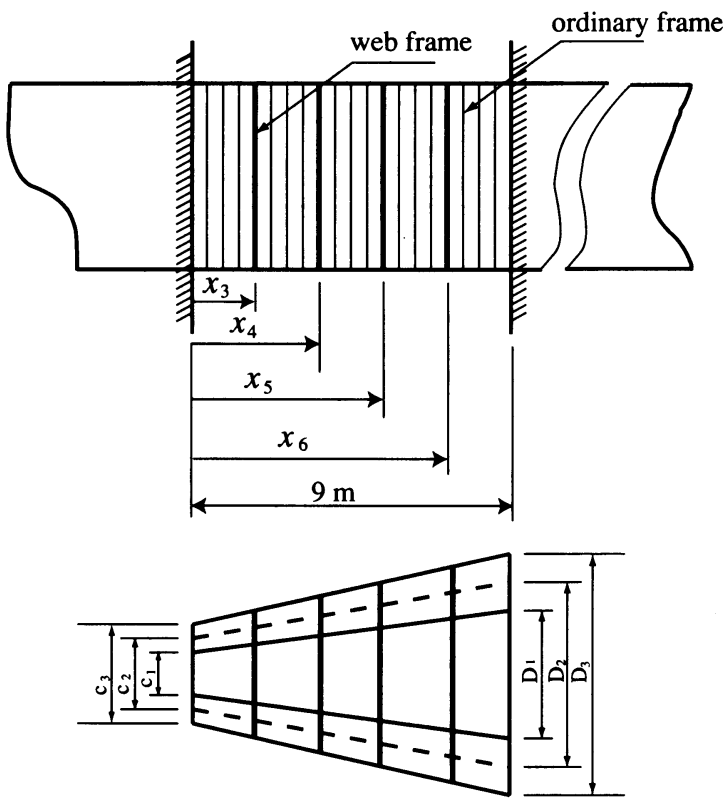

Fig. 7 Geometric change of elements with frame spacing variables 
Table 2 History of the GA

\begin{tabular}{|l|l|l|l|l|l|l|}
\hline Gen & cost & $\sigma_{\max }^{\mathrm{w}}$ & $\sigma_{\max }^{\mathrm{L}}$ & \multicolumn{2}{|c|}{$\mathrm{f}(\mathrm{Hz})$} & $\mathrm{Nm}$ \\
\hline No & $\begin{array}{c}\left(10^{3}\right) \\
\text { yens }\end{array}$ & $\mathrm{kgf} / \mathrm{mm}^{2}$ & $\mathrm{kgf} / \mathrm{mm}^{2}$ & $\mathrm{f}_{13}$ & $\mathrm{f}_{14}$ & \\
\hline 1 & 6789 & 11.55 & 6.360 & 15.408 & 21.323 & 15 \\
3 & 6706 & 13.34 & 6.016 & 15.406 & 21.247 & 11 \\
5 & 5360 & 15.67 & 5.830 & 15.405 & 21.120 & 8 \\
7 & 6116 & 16.83 & 5.413 & 15.403 & 21.104 & 10 \\
12 & 6090 & 16.92 & 5.076 & 15.402 & 21.092 & 7 \\
20 & 6057 & 17.01 & 5.015 & 15.400 & 21.082 & 14 \\
30 & 6043 & 17.11 & 5.018 & 15.400 & 21.083 & 8 \\
35 & 6015 & 17.20 & 5.027 & 15.400 & 21.084 & 10 \\
40 & 6015 & 17.20 & 5.027 & 15.400 & 21.084 & 13 \\
45 & 6000 & 17.23 & 5.031 & 15.400 & 21.084 & 15 \\
\hline
\end{tabular}

Gen No. : generation number

$\mathrm{Nm}$ number of chromosomes satisfying all constraints

$\sigma_{\max }^{\mathrm{w}}: \max$ bending stress of transverse elements

$\sigma_{\max }^{\mathrm{L}}: \max$ bending stress of longitudinal elements

Table 3 Evolution of Design Variables

\begin{tabular}{|r|c|c|c|c|c|c|c|}
\hline Gen & $x_{1}$ & $x_{2}$ & $x_{3}$ & $x_{4}$ & $x_{5}$ & $x_{6}$ & $x_{7}$ \\
\hline No & No & No & $\mathrm{cm}$ & $\mathrm{cm}$ & $\mathrm{cm}$ & $\mathrm{cm}$ & $\mathrm{cm}$ \\
\hline 1 & 20 & 31 & 228 & 339 & 599 & 724 & 11 \\
3 & 18 & 27 & 225 & 342 & 578 & 731 & 11 \\
5 & 13 & 24 & 220 & 312 & 574 & 717 & 10 \\
7 & 6 & 21 & 223 & 338 & 572 & 729 & 10 \\
12 & 5 & 21 & 180 & 315 & 543 & 718 & 10 \\
19 & 4 & 21 & 177 & 326 & 542 & 701 & 10 \\
30 & 4 & 21 & 160 & 344 & 539 & 699 & 10 \\
35 & 4 & 21 & 158 & 353 & 517 & 695 & 10 \\
40 & 4 & 21 & 158 & 353 & 517 & 695 & 10 \\
45 & 4 & 21 & 158 & 356 & 496 & 695 & 10 \\
\hline
\end{tabular}

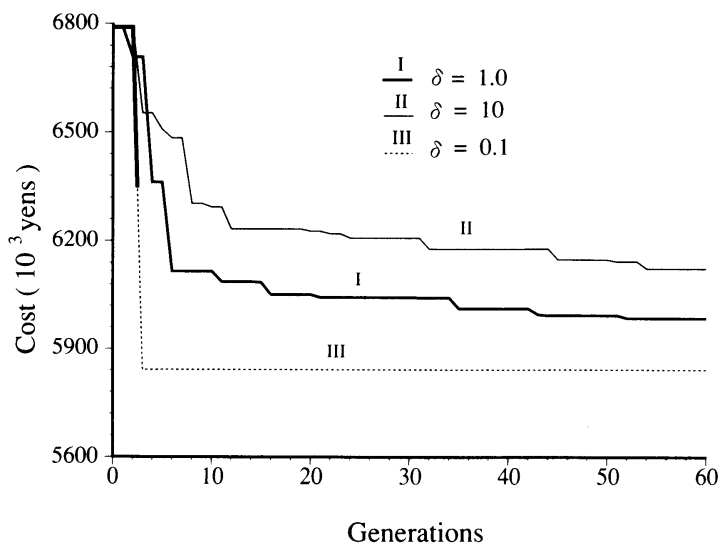

Fig. 8 History of engine room optimization
Fig. 8 shows the influence of the penalty term on the optimization process. Curve II converges prematurely because the penalty terms are too large compared with the objective values. The chromosomes in infeasible domain die out with the generations quickly. A few super chromosomes dominate the process. Curve III represents the results of the GA with very small penalty terms. The dotted part of it means the solutions are not in feasible domain. After the second generation, all the chromosomes are driven into the infeasible domain. The process of the GA ignores the violations of the constraints because the penalty terms are too small.

\section{3. 3 Objective Elitism}

In Fig. 9, $p \circ p^{-}$size $=20, p_{c}=0.7, p_{m}=0.01$, the fitness elitism is introduced for both curve I and curve II. Besides the fitness elitism, for curve I, objective elitism is introduced. The figure shows that the objective elitism improves the convergence significantly. From curve II, it is observed that objective oscillates, especially when the cost value near the optimal value. From the same curve, that the objective value converges to a constant value in general trend can be also observed.

\subsection{Influence of Population}

The influence of population can be observed in Fig. 10 in which $p_{c}=0.7, p_{m}=0.01$, both the fitness elitism and objective elitism are introduced. Obviously curve I terminates prematurely. In this study, it seems that at least 20 chromosomes in one generation is required for this structural optimization.

\section{3.5 Varying Probability of Mutation}

The effect of varying probability of mutation on the performance of the GA is shown in Fig. 11 in which $p_{c}$ $=0.7$, $p o p-$ size $=20$, both the fitness elitism and objective elitism are introduced. Curve I converges quickly at first, but after several generations, curve II is superior to curve I. At the beginning of the optimization process, for curve II, the mutation rate is high, therefore the convergence is not as fast as for curve I.

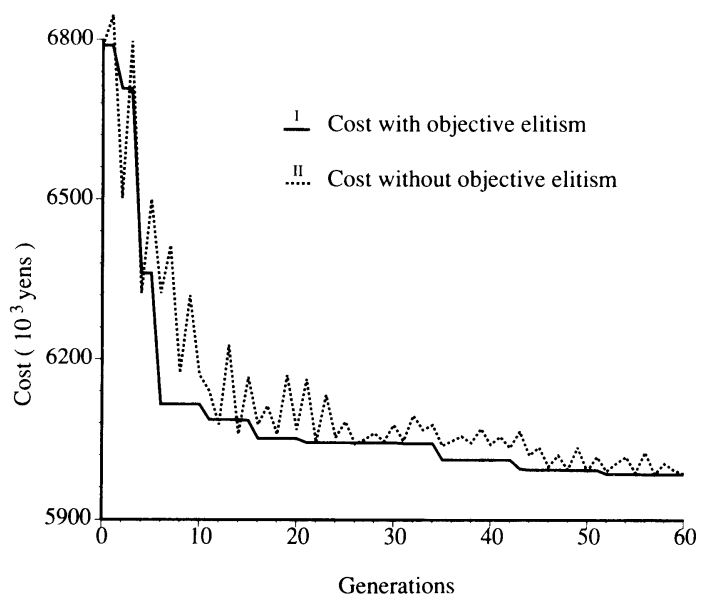

Fig. 9 Comparison of cost between I and II 


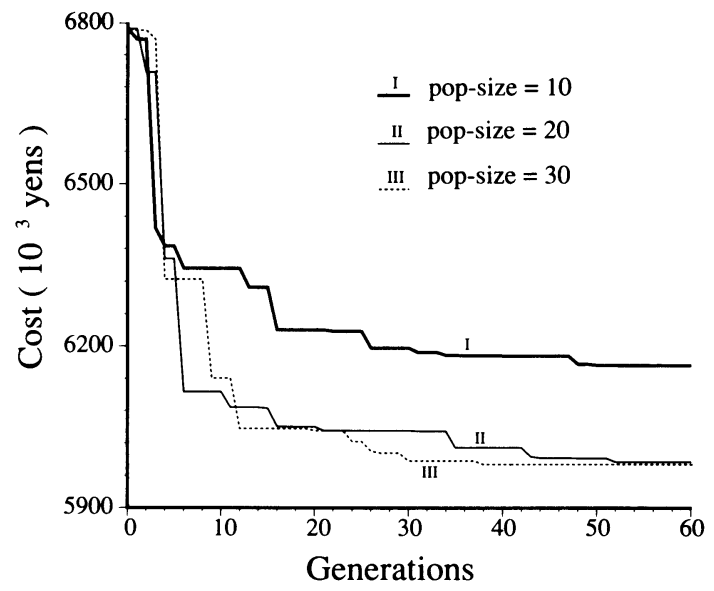

Fig. 10 Influence of population

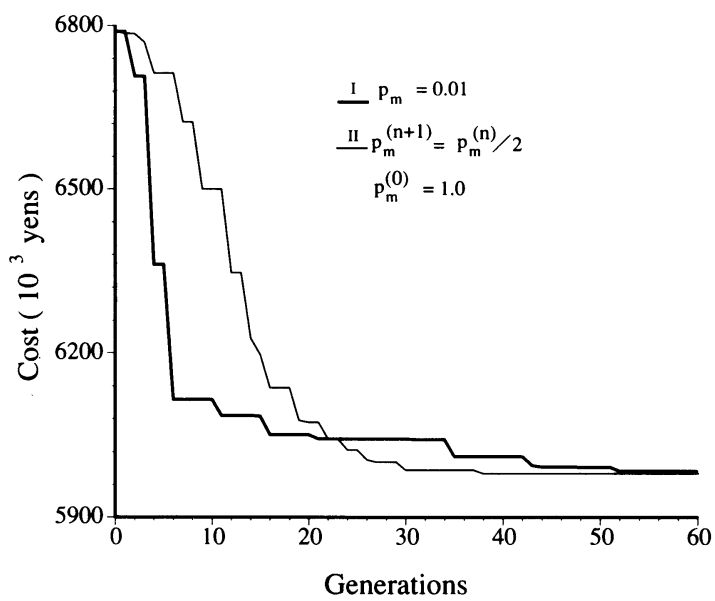

Fig. 11 Influence of probability of mutation

However, because the exploration of curve II is more sufficient than that of curve $I$, it converges faster than curve I later. The objective value of curve II converges to the optimal value at about generation 30 , instead that of curve I converges at about generation 43.

Generally speaking, the model with mutation rate varying slowly contributes to obtaining the global optimal results but its convergence is slower. However, varying the mutation rate too sharply may result in premature even though these kinds of models have the possibility to reach the optimal values rapidly.

6. 3. 6 Crossover Probability $p_{c}$

In this study, eight trials with $p_{c}=0.3,0.4, \cdots, 1.0$ are investigated. The case of $p_{c}=0.3,0.4,0.5,0.6$ converges prematurely. The other cases all converge to the optimal value. For this problem, $p_{c}=0.7$ is the best probability of crossover, with which the GA reaches the objective minimum at generation 43 , others at about generation 55 .

\section{Conclusions}

1) The Genetic Algorithm was used in structural optimization of engine room considering the static and dynamic constraints successfully. The calculation is simple and the search can reach the global optimum if the parameters of the GAs are selected properly.

2) The introduction of the serial numbers makes us do the ship optimization more effectively. Generally speaking, the irregular discrete design variables can be any kind of variables with multiple parameters.

3) The penalty method to handle constraints which are implicit function of design variables is effective. The experience of determining the values of parameters has significance to other real structural optimization problems.

4) The fitness elitism and objective elitism, especially the latter one, improve the Genetic Algorithm for this structural optimization significantly. The introduction of objective elitism makes us set the termination condition of the Genetic Algorithm easily. The proposed termination condition makes the algorithm terminate at certain precision defined previously.

5) The introduction of the varying mutation rate also improves the algorithm significantly. However, some parameters such as the crossover rate, the precision and the nature of objective function may influence the selection of the best varying model. For this problem, $p_{m}^{(n+1)}=p_{m}^{(n)} / 2$ works well.

\section{References}

1) Tetsuo Okada, Isao Neki, Optimization of Ship Structural Design by Genetic Algorithm, J. Soc. Naval Arch. of Japan, (April, 1992), pp 259-266

2) Isao Neki, Tetsuo Okada, Optimum Structural Design Using Genetic Algorithm and Finite Element Method, J. Soc. Naval Arch. of Japan, (July, 1995) pp 327-337

3) Goldberg, D. E., Genetic Algorithms in Search, Optimization and Machine Learning, Addison Wesley, Reading, Mass., 1989

4) Guoqiang Zhou, Hisashi Nobukawa and Fengxiang Yang, Discrete Optimization of Ship Structures from the Viewpoint of Practical Design, J. Soc. Naval Arch. of Japan, (Nov. 1997), pp 551559.

5) Zbigniew Michalewicz, Genetic Algorithms+ Data Structures =Evolution Programs, 3rd rev. Springer-Verlag. 1996

6) Goldberg, D. E., Optimal Initial Population Size for Binary-Coded Genetic Algorithms. Proceeding of the International Joint Conference on Artificial Intelligence. 9, (1985), pp 588-592.

7) Michalewicz, Z. and Janikow, C., Handling Constraints in Genetic Algorithms, Proceeding of the Fourth International Conference on Genetic Algorithms, (1991), pp 151-157.

8) Mitsuo Gen, Runwei Cheng, Genetic Algorithms and Engineering Design, JOHN WILEY \& SONS, INC. 1997 
9) Hisashi Nobukawa, Guoqiang Zhou, Discrete Optimization of Ship Structures with Genetic
Algorithms, Journal of The Society of Naval Architects of Japan, Vol. 179, (1996), pp 293-301 